measure. Let $L$ be a positive number and let $z(t, L)$ be equal either to $z_{1}(t)$ or $z_{2}(t)$ according as $[t / L]$ is even or odd. It is clear that $z(t, L)$ is an S.a.p. solution of $\Phi[z(t), F(t)]=0$ for each positive $L$, and that all these solutions are essentially distinct. Of course many other solutions could be constructed in a similar way.

Brown University and Princeton University

\title{
ON A LEMMA OF FEJÉR*
}

BY LINCOLN LA PAZ

1. Simple Integrals. In an important paper L. Fejér † has verified and used the following lemma.

Lemma A. If for a problem of minimizing an integral

$$
I_{1}=\int_{x_{1}}^{x_{2}} \phi\left(y^{\prime}\right) \cdot f(x, y) d x,
$$

the Euler equation in normal form is

$$
y^{\prime \prime}=F\left(x, y, y^{\prime}\right),
$$

then for a problem of minimizing the integral

$$
J_{1}=\int_{x_{1}}^{x_{2}}\left[\phi\left(y^{\prime}\right) / f(x, y)\right] d x,
$$

the Euler equation in normal form is

$$
y^{\prime \prime}=-F\left(x, y, y^{\prime}\right) \text {. }
$$

The following generalization of Fejér's lemma is proved in this note.

* Presented to the Society, December 2, 1933.

$\dagger$ L. Fejér, Das Ostwaldsche Prinzip in der Mechanik, Mathematische Annalen, vol. 61 (1905), p. 432.

$\ddagger$ In everything that follows, the range of the indices $i, j, k, \mu, \nu$ is from 1 to $n$ and $\mu$ and $\nu$ are umbral. 
Lемма B. If for a non-singular problem of minimizing an integral

$$
I_{n}=\int_{x_{1}}^{x_{2}} \phi\left(y_{1}^{\prime}, \cdots, y_{n}^{\prime}\right) f\left(x, y_{1}, \cdots, y_{n}\right) d x
$$

with integrand function different from zero and of class ${ }^{*} C^{\prime \prime}$, the system of Euler equations in normal form is

$$
y_{k}^{\prime \prime}=F_{k}\left(x, y_{1}, \cdots, y_{n}, y_{1}^{\prime}, \cdots, y_{n}^{\prime}\right),
$$

then for a problem of minimizing the integral

$$
J_{n}=\int_{x_{1}}^{x_{2}} \phi\left(y_{1}^{\prime}, \cdots, y_{n}^{\prime}\right) \Phi(f) d x,
$$

where $\Phi$ is different from zero and of class $C^{\prime \prime}$, but is otherwise an arbitrary function of its argument, the system of Euler equations in normal form is

$$
y_{k}^{\prime \prime}=\left(f \Phi^{\prime} / \Phi\right) F_{k}\left(x, y_{1}, \cdots, y_{n}, y_{1}^{\prime}, \cdots, y_{n}{ }^{\prime}\right) .
$$

In $\left(4^{\prime}\right), \Phi^{\prime}$ denotes the derivative of $\Phi$ with respect to its argument.

This lemma is an immediate consequence of the fact that the Euler systems of the problems (3) and (4) can be put into simple normal forms in terms of the transversality coefficients $t_{i}=$ $\left(\partial \phi / \partial y_{i}{ }^{\prime}\right) /\left(\phi-y_{\mu}{ }^{\prime} \partial \phi / \partial y_{\mu}{ }^{\prime}\right)$ of these problems. $\dagger$ In fact, the Euler equations of (3) and (4) can be written $y_{k}^{\prime \prime}=d_{k} / D$ and $y_{k}{ }^{\prime \prime}$ $=D_{k} / D$, respectively. Here $D=\left|\partial t_{i} / \partial y_{j}{ }^{\prime}\right|$ and $d_{k}$ and $D_{k}$ are the determinants obtained from $D$ by replacing the element $\left(\partial t_{i} / \partial y_{k}^{\prime}\right)$ by

$$
\left[\partial(\log \Phi) / \partial y_{i}-t_{i} \partial(\log \Phi) / \partial x\right]\left(1+y_{\mu}^{\prime} t_{\mu}\right)
$$

* The term class is used in the sense of Bolza, Vorlesungen über Variationsrechnung, pp. 13-14. As C. Carathéodory has recently pointed out (Bermerkung über die Eulerschen Differentialgleichungen der Variationsrechnung, Nachrichten von der Gesellschaft der Wissenschaften zu Göttingen, Mathematisch-Physikalische Klasse (1931), p. 40) the classical assumption that the integrand function is of class $\mathrm{C}^{(n)}, n \geqq 3$, is unnecessary.

$\dagger \mathrm{L} . \mathrm{La} \mathrm{Paz}$, The Euler equations of problems of the calculus of variations with prescribed transversality coefficients, Proceedings of the National Academy of Sciences, vol. 17 (1931), p. 471, (17). 
with $\Phi=f$ and $\Phi=\Phi(f)$, respectively. Since $D_{k}=f\left(\Phi^{\prime} / \Phi\right) d_{k}$, $(k)$, the lemma follows.*

2. Application to Brachistochronic Integrals. As an application of the lemma just established, consider the problem of obtaining a normal Euler system for the important class of brachistochronic integrals in $\left(x, y_{1}, \cdots, y_{n}\right)$-space

$$
T=\int_{s_{1}}^{s_{2}} \frac{d s}{v(r)}=\int_{x_{1}}^{x_{2}} \frac{\left(1+y_{\mu}^{\prime} y_{\mu}^{\prime}\right)^{1 / 2}}{v\left(\left[x^{2}+y_{\mu} y_{\mu}\right]^{1 / 2}\right)} d x,
$$

the extremals of which may be interpreted as the trajectories of light corpuscles moving through a medium in which the velocity of propagation, $v$, depends alone on the distance from a fixed center or, under suitable assumptions, as the paths of seismic rays in the interior of the earth. Examination of the values assigned to $v(r)$, for example by Kummer in his classical paper on astronomical refraction $\dagger$ or by Kövesligethy, Wiechert, and others in connection with the problem of the seismic brachistochrone, $\ddagger$ will show that even in the case $n=1$ the direct determination of normal Euler equations for problems of the form (5) may be a matter of tedious calculation.

However, if we note that when $v(r)=r^{-1}$ the normal Euler system for (13) has the simple form

$$
y_{i}^{\prime \prime}=r^{-2}\left(1+y_{\mu}^{\prime} y_{\mu}^{\prime}\right)\left(y_{i}-x y_{i}^{\prime}\right), \quad(i=1,2, \cdots, n),
$$

then the generalized lemma shows that the normal Euler system when $v=v(r)$ is simply

$$
y_{i}^{\prime \prime}=-\left[v^{\prime}(r) / r v(r)\right] B_{i}\left(x, y_{1}, \cdots, y_{n}, y_{1}^{\prime}, \cdots, y_{n}^{\prime}\right),
$$

where $B_{i} \equiv\left(1+y_{\mu}{ }^{\prime} y_{\mu}{ }^{\prime}\right)\left(y_{i}-x y_{i}{ }^{\prime}\right)$ and $i=1,2, \cdots, n . \S$

3. Multiple Integrals. For $n$-fold integrals of the form

* The lemma may also be established by direct calculation of the Euler equations of (3) and (4) and subsequent solution for the derivatives $y_{j}{ }^{\prime \prime}$.

$\dagger$ E. E. Kummer, Über atmosphärische Strahlenbrechung, Journal für Mathematik, vol. 61 (1863), p. 263.

¥ See, for example, V. Conrad, Dynamische Geologie, Encyklopädie der Mathematische Wissenschaften, vol. VI, 1, 11, 10, p. 453.

$\S$ Compare L. La Paz, loc. cit., p. 461, (19). 


$$
\int_{(n)} f\left(x_{1}, \cdots, x_{n}, z, p_{1}, \cdots, p_{n}\right) d x_{1} \cdots d x_{n},
$$

we can state a result similar to that embodied in the generalized lemma of Fejér. The Euler-Lagrange partial differential equation for a problem of minimizing an integral (8) is

$$
f_{p_{\mu} x_{\mu}}+f_{p_{\mu} z} p_{\mu}+f_{p_{\mu} p_{\nu}} p_{\mu \nu}-f_{z}=0,
$$

where $p_{i}=\partial z / \partial x_{i}$ and $p_{i j}=\partial^{2} z / \partial x_{i} \partial x_{j}$. Consider now two integrals (8) with integrand functions of the special forms

$$
\begin{aligned}
& f_{1}=a\left(x_{1}, \cdots, x_{n}, z\right) b\left(p_{1}, \cdots, p_{n}\right), \\
& f_{2}=\Phi(a) b\left(p_{1}, \cdots, p_{n}\right),
\end{aligned}
$$

where $a, b$ and $\Phi$ are different from zero and of class $C^{\prime \prime}$, but are otherwise arbitrary functions. We then have the following lemma.

LEMмA C. If the Euler-Lagrange equation (9) of a regular problem of minimizing an integral (8) with integrand function $f=f_{1}$ is expressed in the form

$$
b_{p_{\mu} p_{\nu}} p_{\mu \nu}=F\left(x_{1}, \cdots, x_{n}, z, p_{1}, \cdots, p_{n}\right),
$$

then the Euler-Lagrange equation of a similar problem with integrand function $f=f_{2}$ is expressible in the form

$$
b_{p_{\mu} p_{\nu}} p_{\mu \nu}=\left(a \Phi^{\prime} / \Phi\right) F\left(x_{1}, \cdots, x_{n}, z, p_{1}, \cdots, p_{n}\right) .
$$

This result can be justified by an argument based on considerations analogous to those employed in the first section or by a direct verification which can be made under even less restrictive hypotheses than those of the lemma.

The Ohio State University 